\title{
LE BLOC-NOTES EN EXPANSION - AUTRE MEDIA, AUTRE DISCOURS
}

\begin{abstract}
Jereczek-Lipińska Joanna, Le bloc-notes en expansion - autre média, autre discours [The blog expanding - another media, another discourse]. Studia Romanica Posnaniensia, Adam Mickiewicz University Press, Poznań, vol. XXXIII : 2006, pp. 133-143. ISBN 83-232-1643-6, ISSN 0137-2475.
\end{abstract}

This text falls under research of the discursive analysis as in what one qualifies of the blogoscopy to knowing in the analysis still little developed phenomenon of the blogs. These new media are in full expansion in France. We thus propose to analyze this advent of the new media while starting with simple observation of the language which it generates, while passing by its definition, typology and structure and to arrive finally at the influence and the range that it will be able to have on the political and media speech.

Ce texte s'inscrit dans les recherches de l'analyse discursive ainsi que dans ce que l'on qualifie de blogoscopie à savoir dans l'étude encore peu développée du phénomène des blogs. Ce nouveau média acquiert tout doucement le droit de cité dans le paysage médiatique français. Nous nous proposons ainsi d'analyser cet avènement du nouveau média en commençant par simple observation du langage qu'il génère, en passant par sa définition, typologie et évolution et pour en arriver enfin à l'influence et la portée qu'il pourra avoir sur le discours politique et médiatique.

\section{CE VOCABULAIRE QUI EN DIT BEAUCOUP SUR LE PHÉNOMĖNE}

Il sera question ici de toutes ces pages personnalisées Web étant une sorte de journal de bord qui se présentent sous la forme de carnets en ligne. Les initiés intemautes y livrent plus ou moins régulièrement leurs posts, datés et avec de nombreux liens vers d'autres sites.

Le blog est, tout le monde en convient, un média à part entière, un média nouveau. Et en tant que tel, il génère un langage censé refléter la réalité qui s'y attache. Passons justement en revue quelques mots voire notions nouvelles qui en 
disent beaucoup sur le phénomène même. A commencer par le terme de blog (et ses nombreuses variantes: weblog, joueb, blogue) qui vient de l'anglais car le concept nous vient des Etats-Unis de la fin de l'année 1997. Mais il s'est vraiment développé après le 11 septembre 2001 comme réaction à l'attentat. En France les tout premiers blogs ont fait leur apparition en 2004 lors des régionales. Le blog est récent dans la réalité des médias français, la preuve en est que le mot ne se trouve pas encore dans les dictionnaires de référence. Le Petit Robert et Le Larousse prévoient son entrée dans l'édition 2006 seulement.

La provenance du phénomène fait que nous avons affaire dans cette terminologie, à de nombreux anglicismes, de multiples néologismes en franglais, l'omniprésence de technolectes en anglais francisé et déjà quelques équivalents francophones.

C'est un langage très expressif et surtout dynamique dans sa créativité dérivationnelle et compositionnelle. Nous avons ainsi toute une série de dérivés qui continuent à en créer d'autres encore. La personne qui ouvre son blog (blogue) est qualifiée de blogueur ou blogueuse (soit bloggers ou encore blogers - vu la nouveauté du phénomène, on verra plus d'une fois que l'orthographe est hésitante et floue mais l'usage finira par se stabiliser). Pour l'instant, il faut admettre que ce langage est souple au point d'offrir à l'usager tout un éventail de termes qui désignent bien souvent la même réalité, ainsi nous rajoutons encore à la liste précédente - le billettiste qui est une autre appellation de celui qui publie son blog. Les blogueurs bloguent tout ce qui est blogable. Autrement dit, il n'y a pas de contraintes à ce sujet, par contre on blogue sur tout ce qui intéresse le blogueur, et est censé intéresser son public. Ceci relève d'ailleurs de la blogoréalité. C'est un fonctionnement classique d'un média traditionnel et pourtant la différence de taille se fait sentir, nous y reviendrons ultérieurement. L'action de bloguer et de lire est le blogage. Et faire paraître les textes donc les posts sur un blog, c'est les y poster. En fonction de la régularité des blogueurs, nous avons à notre disposition des métaphores plus ou moins réussies, ainsi la blogorrhée indique qu'on blogue d'une manière très régulière voire quotidiennement ou la blogostipation qui met en relief la difficulté à suivre ses textes.

Tout blogueur fait partie de la blogosphère (parallèlement nous avons la papiosphère).

D'ailleurs, c'est une véritable communauté, assez soudée, qui se réfèrent régulièrement à elle-même. Tous les blogueurs, dans chacun de leurs posts citent en effet d'autres blogs et affichent une liste de leurs liens préférés. Les blogs sont ainsi une sorte de bibliothèque de liens et ces listes de liens portent le nom de blogolistes. En outre, ceux qui atteignent le plus grand audimat font partie de la blogogeoisie (ou blogeoisie), le terme a d'ailleurs pris, entre temps, une teinte ironique. Nous rencontrons d'ailleurs plus d'une fois les dérivés selon les registres différents notamment pour blogosphère, nous avons déjà d'un côté blogobile (marqué 
ironique) et de l'autre blogoboule (marqué familier). En tout cas nous avons affaire à un pullulement de termes néologiques blogosphèriques.

Comme la blogosphère a pris une couleur péjorative, un autre terme se crée tout en se spécialisant - les V.I.B. (very important blogger). Ce sont les blogs de référence qui donc parviennent à se démarquer.

La terminologie des blocs-notes varie selon les pays francophones même si le phénomène est essentiellement virtuel donc allant au-delà des frontières qu'elles soient linguistiques ou géographiques. Ainsi, dans la version québécoise, nous avons de multiples équivalents francophones, ainsi le blog devient cybercarnet, carnet ou carnet Web, ses auteurs - carnetiers (carnetières) ou carnétistes (au féminin - carnettistes) et ils appartiennent tous au domaine de la carnetosphère. Et nous ne bloguons point, nous carnetisons.

La terminologie blogosphérique, récente qu'elle soit, suit son cours naturel de l'évolution et de l'expressivité. Or, La Commission Générale de Terminologie et de Néologie veille et tente de maîtriser la situation qui lui échappe quelque peu. Dans son « Journal officiel » du 20 mai 2005 , dans la rubrique Vocabulaire de l'Internet (liste de termes, expressions et définitions adoptés) nous pouvons lire ce qui suit:

\section{bloc-notes, n.m.}

Forme abrégée : bloc, n.m.

Domaine : Informatique/Internet.

Définition : Site sur la toile, souvent personnel, présentant en ordre chronologique de courts articles ou notes, généralement accompagnés de liens vers d'autres sites.

Note : La publication de ces notes est généralement facilitée par l'emploi d'un logiciel spécialisé qui met en forme le texte et les illustrations, construit des archives, offre des moyens de recherche et accueille les commentaires d'autres internautes.

Équivalent étranger : blog, web log, weblog. (http:/www.culture.gouv.fr/culture/dglf/dispositifenrichissement.htm)

La Commission a choisi un terme existant et qui signifie «bloc de papier pour prendre des notes " (Le Petit Robert, 2000). Reste à savoir s'il est vraiment pertinent par rapport à la réalité qu'il est censé exprimer. Mais surtout elle se prononce implicitement sur l'omniprésence de l'anglais dans ce phénomène, en recommandant le terme de bloc-notes et en abrégé de bloc plutôt que le blog qu'elle considère comme étant étranger. Dans cette optique là, il appartient de citer ici un journaliste du Service politique du Nouvel Observateur - Robert Schneider qui a ouvert son blog-notes pour jouer voire jongler avec cet anglais francisé (http://blogs.nouvelobs.com/Robert_Schneider/index.php).

Comme on peut le voir à travers cette multitude de néologismes, ce langage est très productif. Il a et continue à prendre une ampleur rapide et imprévisible ainsi qu'une allure d'un parler jeune, dynamique, branché. Et avec ses mots courts et à mi-chemin entre l'anglais et le français, il se situe au rang des argots du Net.

Il nous importe d'admettre, qu'il existe bel et bien des milliers de blogs et ceux-ci, de qualité et d'intérêt très variables. D'où notre décision d'écarter de notre 
analyse les blocs des adolescents ou autres citoyens lambda, où nous avons surtout affaire à une confusion de genres due plus au hasard que véritablement à une caractéristique du style (c'est une caractéristique du langage d'une génération à un moment donné de leur vie). Par contre, nous avons choisi de travailler sur les V.I.B. à savoir les blocs des politiques et journalistes réputés qui nous paraissent plus fiables et représentatifs de la spécificité du genre en question sur le long terme. Nous allons donc retracer ce qui relève de la bloguitude considérée comme ensemble de traits caractéristiques propres aux blogs.

\section{DE LA PAPIOSPHÈRE A LA BLOGOSPHÈRE}

Le blog est un média (pas) comme les autres. Le problème est loin d'être résolu. En effet, les uns le considèrent comme média alternatif et donc en concurrence avec d'autres médias, les autres comme média complémentaire et d'autres encore vont jusqu'à dire que les blogs sont un dispositif à part, une sorte de portevoix personnel et virtuel marquant ce début du siècle. La question est de savoir quelle est la spécificité des blocs face aux autres médias tout en sachant que le blog en tant que phénomène récent, se prête difficilement à des conclusions définitives. Nous assistons à sa phase de transition seulement et tout constat ne peut concerner que cet outil à cette étape de son fonctionnement. Avant d'étudier ses formes d'expression, nous nous proposons de dégager ses principales caractéristiques qui font ses succès en dépit d'autres instruments médiatiques.

Pour cerner au mieux le phénomène, nous avons fait le tour d'opinions du côté des blogueurs. Les nombreux utilisateurs des blocs les envisagent en terme de journal intime, dialogue permanent, livre ouvert ou encore calepin virtuel. Il faut admettre que chacune de ces expressions met en relief une de ces caractéristiques principales à savoir, c'est de l'écriture personnelle voire personnalisée, interactive et infinie. C'est un journal de bord en ligne direct, participatif et mis à jour plus ou moins systématiquement.

Nous pouvons déjà distinguer une typologie de blogs. Premièrement, les blocs introspectifs, une sorte de journal intime tenu à jour par un particulier, par exemple ceux des adolescents avides d'expression et de se faire connaître et de se faire reconnaître (les lycéens se plaignant de leurs professeurs en est un cas parmi d'autres) ou encore les blogs des citoyens lambda comme par exemple celui d'une femme au foyer. Viennent ensuite les blogs d'opinion (qui constituent l'objet de cette recherche) - tenus par des journalistes professionnels ou politiques. Ils analysent l'actualité sous un angle social, économique, politique ou satirique. Le troisième groupe est constitué par les blogs pro/techno - une sorte de métablog où nous pouvons trouver des informations sur le média en question. Et enfin les blogs à part - satiriques, souvent créatifs et originaux, en tout cas, difficiles à définir comme c'est le cas de ce bloc très intéressant - http:/www.wmaker.net/julo/index.php. 
Revenons donc à ce qui nous préoccupe ici à savoir la blogosphère politique et médiatique. En France, la campagne des régionales 2004 a vu naître le phénomène des blogs en politique. Alain Rousset (PS), André Santini (UDF) puis Jean-François Copé (UMP) ont ouvert leurs blogs comme un véritable support de campagne. Dominique Strauss Kahn fait lui aussi partie des précurseurs, mais ses objectifs étaient repartis sur le long terme - «J'ai souhaité lancer ce forum pour pouvoir dialoguer directement avec tous ceux qui veulent participer au débat public. (...) Vous pouvez d'ores et déjà réagir sur des thèmes comme la justice, la recherche, les relations transatlantiques, la campagne des régionales et des cantonales... J'attends toutes vos suggestions ! " (http://www.blogdsk.net/) Ainsi, au sein des blogs politiques, nous pouvons distinguer quelques catégories. Ceux qui n'apparaissent que pour servir la campagne électorale ou référendaire (http://www.aquitainerousset.net/, le oui/non pour le Traité de Constitution - http:/www.oui-et-non.com/) et ceux qui résistent aux temps et continuent à sillonner la carrière politique du politique auteur du blog indépendamment des fonctions qu'il exerce ou qu'il n'exerce plus, comme c'est le cas d'Alain Juppé (http://www.alljup.com/).

En ce qui concerne les blogs des joumalistes, nous avons relevé une structure, une sorte de moule dans lequel ils mettent leurs idées souvent celles qu'ils ne peuvent exprimer, pour plusieurs raisons de contraintes ou autres, ailleurs. L'exemple significatif à cet égard est le blog des correcteurs du Monde - Martine Rousseau et Olivier Houdart (http://correcteurs.blog.lemonde.fr/) où ils expliquent les corrections administrées lors de leur travail mais qu'ils n'ont pas pu jusqu'à là expliciter en public.

Le blog politique permet le débat permanent, offre également aux auteurs la possibilité de tester leurs idées. Il leur permet de se rapprocher de leur audience et de leurs partisans ainsi que de passer d'un discours institutionnel à un ton plus personnel. Cela signifie que l'on attend du blogueur qu'au lieu d'évoquer les grandes idées politiques, il exprime le point de vue privé de l'homme public. Le blog des journalistes vise d'autres objectifs et parmi eux l'envie d'échanger et de partager leurs lectures de l'actualité.

Une remarque s'impose ici, si nous avons distingué le blog d'un politique et d'un joumaliste de celui d'internautes lambdas, c'est tout aussi parce que l'un et l'autre, en tant que personne publique ont généralement à leur disposition un ensemble de moyens pour communiquer (presse du parti, télévision, radio, site Internet ...) et le blog apparait alors comme un outil supplémentaire. L'attention devrait se porter sur ce qui, pour l'homme politique ou pour journaliste paraît le plus inédit. Autrement dit, l'exploitation de la structure - blog pour simplement diffuser une information traditionnelle ne présente pas une révolution dans la pratique médiatique ou politique (tel est le cas du blog de François Hollande - http://www.fhollande.net/ qui ne fait que retransmettre en ligne les discours prononcés ailleurs). En revanche, s'il est le support à un nouveau type de message aussi bien dans sa forme que dans son contenu, nous pouvons alors estimer que le défi a été relevé. 
Car il convient de souligner ici que le rôle du weblog n'est pas de chercher et transmettre l'information. Il la déconstruit, reconstruit, confronte, l'amplifie, la remet en question, la soumet au débat. Ce n'est donc pas tellement une forme différente de journalisme mais un média à part entière. Il serait donc plus juste, d'en parler comme d'une nouvelle pratique qui semble avoir la forme la plus aboutie d'un ensemble de nouveaux dispositifs de communication : chats, forums, messageries, wikis ...

\section{L'ART DU BLOG - LES LOIS DE LA BLOGUÉCRITURE}

Tout nouveau média adopte le langage des médias existant pour en arriver à se forger, avec le temps et en fonction du support, son langage spécifique. Tel sera le cas des blocs-notes, ce qui fait qu'aujourd'hui nous ne pouvons que constater l'évolution dans laquelle s'est engagée cette forme d'expression.

Notons déjà quelques caractéristiques des blocs-notes des politiques et journalistes que nous regroupons ici même si leur langage est considérablement différent. Mais notre but est, pour commencer, de mettre en évidence les caractéristiques du genre et dans cette optique les blogs des uns et des autres présentent des caractéristiques communes. L'étape suivante sera de relever les différences et par là même la spécificité propre à chacun de ces deux groupes.

L'objectif principal de cette forme verbale dans les deux cas, est de se rapprocher du public, d'échanger, c'est une sorte de tac au tac car l'essentiel est qu'il n'y a plus d'intermédiaires - de journalistes pour les politiques ou de différents niveaux de la rédaction pour les journalistes.

\section{(CON)FUSIONS RÈGNENT}

Daniel Schneidermann analyse la situation médiatico-politique en termes d'«irrésistible polyphonie » qu'il explique comme suit « dans l'emballement tous les protagonistes se confondent, ceux qui parlent et ceux qui écoutent, journalistes et lecteurs, témoins et acteurs, tous colportent le même message " (Schneidermann, 2004, 16).

Le blog est une des manières pour tous de s'inviter au débat public. Dans ce contexte, la confusion de genres ou la fusion de genres est de mise. Le blog est une sorte d'interdiscours où les langages de différents médias s'interpénètrent. Ainsi, nous pouvons y observer les caractéristiques de l'article de presse, les traits propres à l'art épistolaire, au courriel ou encore à la conversation en direct :

- l'article de presse - les posts sont regroupés en rubriques titrées, le titre est d'ailleurs un dénominateur commun qui saute le plus aux yeux, en effet, nous retrouvons ici tout le procédé de titrage traditionnel à savoir tous les types de titres 
et leur fonction et la façon de les construire s'apparentent beaucoup à la manière de le faire au sein de la presse traditionnelle - le titre, le sous-titre, le chapeau et moins souvent l'intertitre. En outre, les textes sont découpés en paragraphes, tout ceci pour obtenir la lisibilité et efficacité maximales. Vient ensuite la présentation d'une thématique sous forme du document iconico-textuel. Dans le langage nous remarquons systématiquement le souci de vouloir bien expliquer ce qui amène le blogueur à recourir aux éléments de vulgarisation. Il s'ensuit que la structure des posts présente quelques similitudes évidentes - le titre en haut, le corps du texte et la signature de l'auteur avec un élément en plus qui est l'heure à laquelle le texte est posté ;

- l'art épistolaire, le courriel ou encore la conversation en direct - nous constatons l'emploi des formules d'appel et finales - Bonjour à toutes et à tous, A bientôt, peut-être à demain dsk (http://www.blogdsk.net/, l'utilisation de la fonction phatique - êtes-vous toujours là ? Le contenu est également inhabituel des discours officiels, et que l'on trouve plutôt dans les communications personnelles comme le sujet des vacances - Je m'absente quelques jours pour une réunion de famille (http://delvolve.typepad.com/). Une autre caractéristique qui s'apparente au discours des conversations et à l'art épistolaire est l'anticipation aux éventuelles répliques - vous direz que...

Voici d'autres caractéristiques qui confirment l'une après l'autre ce mélange de genres et de discours. Ainsi tout post est ancré même si (ce qui est rare) il n'y a pas de marques explicites de temps, d'espace ou de personne dans le corps du texte. Car le blogueur poste ses textes qui se positionnent ensuite sur son site avec le nom de son auteur, la date et l'heure de sa rédaction (les plus récents sont en haut) avec une possibilité pour le visiteur de laisser un commentaire. Dans l'immense majorité des cas, les posts s'ancrent à travers l'embrayage car pour évoquer et commenter l'actualité, le carnetier est amené à employer les embrayeurs classiques comme les embrayeurs de personne - le « je » et le «tu » ou le « vous », ainsi que les déictiques d'espace et de temps.

En outre, les blogs en tant que réseau de liens offrent les multiples renvois et un véritable jeu de discours direct et indirect ainsi que modalisation autonymique. Le problème c'est que trop souvent encore la polyphonie s'y fait sentir. Derrière les mots d'un politique ou journaliste, on entend la voix de son parti ou de la rédaction, autrement dit dans beaucoup de cas les voix se confondent.

Autant de pistes à explorer pour déchiffrer ce discours pour en tracer sa spécificité et l'évolution. Car nous en sommes à sa phase de transition seulement. Le vrai discours des blogs n'est pas encore né. Car ils mutent considérablement, nous constatons ces changements au cours de nos recherches. Ils s'adaptent aux besoins ou envies de leurs utilisateurs.

Les posts sur les weblogs sont à mi-chemin entre l'écrit et l'oral, une sorte d'écrit oralisé. D'où de nombreuses ellipses, abréviations et raccourcis. Les phrases 
sont généralement courtes, ce qui est peut-être assez spécifique pour les journalistes, autant pour le politique ceci demande un effort supplémentaire et considérable. Ces mêmes phrases sont d'ailleurs bien souvent inachevées comme si on les interrompait en cours d'une discussion. Souvent d'ailleurs le but en est de laisser l'internaute en suspens. Ce procédé permet également de créer une impression d'être en connivence d'esprit : on n'a pas à tout dire, on se comprend sans mots. Il ne faut pas oublier la ponctuation expressive qui est omniprésente, surtout nombreux sont les points d'exclamation, voici l'exemple d'un couple de deux phrases qui présente presque toutes ces caractéristiques à la fois: On croit rêver ! La directive «service», vigilance ! Nous y trouvons également nombre d'interjections - et bien, bon ben, hein ou autres. Il importe de signaler que ces hommes et femmes publiques se permettent également de jouer avec les mots et sens ou avec des chiffres et lettres comme l'a fait Alain Juppé dans l'appellation de son blog - All Juppé (http://www.al1 jup.com/).

L'idéal serait d'arriver au langage concis, précis, clair, efficace et surtout parlant car la lisibilité, l'urgence et proximité obligent. En effet, le blog n'accepte pas de langue de bois. L'alternative est claire, soit on aborde le sujet et on fait mouche soit on ne le touche pas. Parler pour ne rien dire n'a pas de sens ici, mais la question reste de savoir si c'est toujours parler pour tout dire, ceci laisse encore à désirer.

Parfois le blog relève d'un genre narratif avec de nombreuses descriptions accompagnées de photos ou documents visuels, tel est le cas de quelques bloc-notes des journalistes reporteurs, nous y trouvons le langage imagé et métaphorique.

Les blogueurs recourent justement au vécu de ceux à qui ils s'adressent en employant les références et allusions qui leur sont proches - tel est le cas par exemple de l'expression qui vient des paroles d'une chanson - « la positive attitude $"-\cdots$ les mots d'une chanson continuent à vivre leur vie dans le langage de tous les jours. Ainsi on parle aujourd'hui de Chirac qui garde sa positive attitude. Un autre mot - trouvaille des blogs dans le contexte du référendum est le terme de raffarindum ou encore l'exemple de cette métaphore désormais bien connue et mettant en jeu le plombier polonais. Le blog a repris ainsi la fonction qui appartenait jusqu'à là à la presse et qui consistait à détecter les termes voire expressions néologiques pour les (ré)employer - c'est à partir de là qu'on parlait de l'attestation du mot ou d'une locution. Les journalistes sont considérés comme les plus prompts à aller chercher les nouveautés que la langue n'arrête pas d'introduire - le blog semble être encore plus rapide à cet égard (les oui-ouistes et les nonistes du scrutin référendaire sur le Traité de Constitution en constituent également la preuve).

Le visiteur d'un blog s'attend avant tout à vivre un nouveau mode de relation avec le politique ou le journaliste et donc ceci passe par l'utilisation d'un ton relativement différent de celui que le citoyen peut observer dans les autres médias : plus proche, plus décontracté, plus ouvert. Par sa forme, il doit participer au 
rapprochement entre le politique et le citoyen, encourageant ainsi le débat d'idées et l'effacement des frontières, un peu à la manière d'un café politique dans le monde réel. Il s'en suit que le processus de communication via les blocs n'est pas vertical donc du haut vers le bas mais pour la première fois dans la famille des médias, il y en a un qui permet la communication à l'horizontal et sans intermédiaires.

Outre l'adoption d'un ton plus naturel et direct, il doit également être personnel et impératif.

Le blog ne doit pas se confondre avec le site web du politique, même si son accès peut bien entendu se faire via ce dernier tout comme le blog des journalistes ne doit pas être considéré comme émanant du site du journal qui l'engage même si, encore une fois, l'accès se fait via le site de "Libération », du " Monde » ou du «Nouvel Observateur».

Pour terminer, il faut admettre que les blocs constituent un outil simple de discussions, c'est un langage direct, sans façon, une sorte de parler jeune en tout cas moins officialisé et institutionnel. L'on se rend compte que les styles sont disséqués, perçus, ressentis. Car il s'agit de transmettre beaucoup plus qu'une information, à savoir une sensibilité, un point de vue, une personnalité.

\section{LA FINALITÉ ET CIBLE VISÉE}

L'homme politique devrait bloguer parce que cela le rapproche de son audience et de ses partisans, cela crée un espace - débat, il peut tester et diffuser plus facilement ses idées, passer d'un discours institutionnel à un ton plus personnel, mieux comprendre les critiques, lever des fonds pour sa cause, etc. Le journaliste qui blogue c'est plutôt pour échanger et transmettre ses perceptions de l'actualité tout en se libérant des contraintes du métier.

En terme de finalité des blogs, il faut admettre que c'est comme pour tout média, il s'agit de toucher le plus grand public. Et pour ceci la solution idéale est de bien viser sa cible. Tout blogueur devrait être conscient de sa cible principale et de ses cibles de conquête, tout en sachant que le blog est interactif et pour demander de réagir, il convient de soumettre au public un argument qui le ferait bouger. En effet, Internet est un média participatif.

Pour les blogs en question, la cible est difficilement définissable, s'agirait-il de ces jeunes dynamiques et branchés ou encore des hommes d'affaire, cadres branchés et engagés et enfin d'autres politiques et journalistes et donc des initiés du milieu ou en préférence de tous ces groupes à la fois. On peut supposer que le blog d'un politique va dans un premier temps attirer des visiteurs sympathisants. Cependant, pour attirer de nouveaux publics et ne pas s'enfermer dans des discours d'avertis, le blog ne devrait pas s'adresser qu'aux sympathisants. L'enjeu serait donc de trouver le juste milieu, tant dans la forme que dans le fond, en appréhendant au mieux ceux avec qui on échange. 
La conclusion qui en ressort est qu'il faudra s'adresser à un public non ciblé, ce qui n'a normalement pas lieu d'être en matière de communication. Une philosophie qui persistera tout au long des campagnes : "Si l'un se pose une question, il faut répondre à tous ".

\section{LA PORTÉE DES BLOGS EN COMMUNICATION POLITIQUE ET MÉDIATIQUE}

«Quotidiens, brefs, décalés et engagés, les articles postés sur de nombreux blocs-notes ont de plus en plus de public en quête d'informations pointues et de commentaires acérés » (http://www.loiclemeur.com/france/).

Cet outil de communication est devenu pour beaucoup une source d'informations parallèles et complémentaires aux médias traditionnels qui s'inspirent d'ailleurs également de ce support nouveau. La proximité, l'échange et la connivence que le blog crée avec son public, pourront en faire un véritable instrument de démocratie directe et participative.

Le phénomène étant encore trop récent, il est difficile aujourd'hui d'en tirer les conclusions définitives sur l'impact éventuel qu'aura le bloc sur d'autres médias en présence.

Ce qui est sûr c'est qu'il se fait déjà sa place en se spécialisant comme l'indique celui, qui se veut spécialiste du phénomène, Loïc le Meur - « Les médias traditionnels diffusent des messages, les blogs démarrent des discussions " (http://www.loiclemeur.com/france/).

Il s'agit donc désormais de considérer le blog comme étant l'un des dispositifs dans ce nouvel ordre communicatif.

Prenons à présent, deux événements récents qui ont marqué la politique française dont le traitement sur les blocs-notes nous indiquera leur impact éventuel sur le cours des débats. Pour ce faire nous avons parcouru le traitement qui a été fait sur la blogosphère de l'affaire Gaymard du début de l'année 2005. Le silence significatif des blogueurs sur cette affaire montre plutôt leur limite et marque une véritable victoire de la presse traditionnelle qui s'est vite emparée du phénomène pour en débattre tous les aspects brûlants. Pendant ce temps-là les politiques et même les journalistes sur leurs blogs ont préféré taire cette affaire.

Or, la lecture de la charte du bloggeur, "we the media" (wethe-media. oreilly.com) nous apprend que les bloggeurs ont la possibilité d'orienter la polémique et tel est le cas des blogs aux Etats-Unis où ils ont une réelle influence.

Dans leur grande majorité, les blogs personnels francophones sont encore assez discrets et se limitent à une analyse des faits. Rares sont les blocs qui se lancent dans un commentaire véritablement polémique. Il en résulte que le blog en France n'est pas, pour l'instant du moins, un blog d'investigation, pas un blog d'opinion, 
juste un blog de commentaire, "sorte de chambre d'écho des médias traditionnels » (http://www.loiclemeur.com/france/).

Mais en l'espace de quelques mois à peine, la situation a considérablement changé. Car, lors de la campagne référendaire sur le Traité de Constitution, on a assisté à un changement notable pour le statut des blocs sur le marché des médias. Alors que tous les autres médias faisaient campagne à sens unique pour le " oui », c'est sur les blocs qu'on a vu s'ouvrir un vrai débat de fond et où les arguments "pour » et «contre » pouvaient trouver leur place (http://www.oui-et-non.com/). Et vu le résultat du scrutin, nous ne pouvons plus sous-estimer l'importance de ce dispositif sur les scènes politique et médiatique désormais aussi virtuelles.

\section{BIBLIOGRAPHIE}

Balle F. (2003), Médias et sociétés, Librairie Générale de Droit et de Jurisprudence.

Fiévet C., Turrettini E. (2004), Blog Story, Organisation.

Le Petit Robert (2000), Le Robert, Paris.

Maingueneau D. (2000), Analyser les textes de communication, Nathan Université.

Schneidermann D. (2004), Le cauchemar médiatique, Editions Denoël.

http://www.culture.gouv.fr/culture/dglf/dispositif-enrichissement.htm

http:/www.culture.gouv.fr/culture/dglf/cogeter/publications-jo.htm

http://www. francopee.com/carnet/archives/000229.html

http:/www.wmaker.net/julo/index.php

http:/www.microcontentnews.com/articles/blogosphere.htm

http:/embruns.net/etude_du_blogue/000034.html

http://www.wmaker.net/julo/index.php

Les blogs des politiques

http://lefil.blogs.com/benoithamon/

http://michel-moine.typepad.com/

http://www.blogdsk, net/

http://www.all jup.com/

http://iledefrance.skyblog.com/

http://delvolve.typepad.com/

http://yvesjego.typepad.com/blog/

Les blogs des journalistes

http://correcteurs.blog.lemonde.fr/

http://blogs.nouvelobs.com/Robert_Schneider/index.php

http://blogs.nouvelobs.com/Laurent_Joffrin/

http://education.blogs.liberation.fr/

http://sylvielasserre.blogs.com/ 